\title{
Double Laplace Transform Method for Solving Space and Time Fractional Telegraph Equations
}

\author{
Ranjit R. Dhunde ${ }^{1}$ and G. L. Waghmare ${ }^{2}$ \\ ${ }^{1}$ Department of Mathematics, Datta Meghe Institute of Engineering Technology and Research, Wardha, India \\ ${ }^{2}$ Department of Mathematics, Government Science College, Gadchiroli, India \\ Correspondence should be addressed to Ranjit R. Dhunde; ranjitdhunde@rediffmail.com
}

Received 17 June 2016; Revised 23 September 2016; Accepted 5 October 2016

Academic Editor: Irena Lasiecka

Copyright (c) 2016 R. R. Dhunde and G. L. Waghmare. This is an open access article distributed under the Creative Commons Attribution License, which permits unrestricted use, distribution, and reproduction in any medium, provided the original work is properly cited.

\begin{abstract}
Double Laplace transform method is applied to find exact solutions of linear/nonlinear space-time fractional telegraph equations in terms of Mittag-Leffler functions subject to initial and boundary conditions. Furthermore, we give illustrative examples to demonstrate the efficiency of the method.
\end{abstract}

\section{Introduction}

The telegraph equation developed by Oliver Heaviside in 1880 is widely used in Science and Engineering. Its applications arise in signal analysis for transmission and propagation of electrical signals and also modelling reaction diffusion.

In recent years, great interest has been developed in fractional differential equation because of its frequent appearance in fluid mechanics, mathematical biology, electrochemistry, and physics. A space-time fractional telegraph equation is obtained from the classical telegraph equation by replacing the time and space derivative terms by fractional derivatives.

There are various methods developed to solve fractional telegraph equations. Orsingher and Xuelei [1] and Orsingher and Beghin [2] considered the space and time fractional telegraph equations and obtained the Fourier transform of their fundamental solution. Momani [3] and Garg and Sharma [4] used Adomian decomposition method developed by Adomian in [5] for solving homogeneous and nonhomogeneous space-time fractional telegraph equation. Chen et al. [6] implemented separation of variables method for deriving the analytical solutions of the nonhomogeneous time fractional telegraph equation under Dirichlet, Neumann, and Robin boundary conditions. Huang [7] considered the combine Fourier-Laplace transform to solve time fractional telegraph equation.
Variational iteration method is proposed by $\mathrm{He}[8]$ and used by Sevimlican [9] for solving space and time fractional telegraph equations. Yildirim in [10] used a homotopy perturbation method and Das et al. in [11] used a homotopy analysis method to obtain approximate analytical solution of fractional telegraph equation. Garg et al. [12] and Galue [13] used generalised differential transform method to derive the solution of space-time fractional telegraph equations in terms of Mittag-Leffler functions. Srivastava et al. [14] applied reduced differential transform method to solve Caputo time fractional order hyperbolic telegraph equation.

In recent years, significant attention has been given by many authors towards the study of fractional telegraph equations by using single Laplace transform combined with variational iteration method, homotopy analysis method, and homotopy perturbation method. Khan et al. [15], Kumar et al. [16], and Prakash [17] applied a combination of single Laplace transform and homotopy perturbation method to obtain analytic and approximate solutions of the spacetime fractional telegraph equations. Alawad et al. [18] used a combination of single Laplace transform and variational iteration method for finding exact solutions of space-time fractional telegraph equations in terms of Mittag-Leffler functions. Kumar in [19] coupled single Laplace transform and homotopy analysis method for the solution of spacefractional telegraph equation. 
To our knowledge, solving fractional partial differential equations using the double Laplace transform is still seen in very little proportionate or no work is available in the literature. So, the main objective of this paper is to find the exact solutions of homogeneous and nonhomogeneous space-time fractional telegraph equations in terms of MittagLeffler functions subject to initial and boundary conditions, by means of double Laplace transform.

\section{A Brief Introduction of}

\section{Double Laplace Transform and} Caputo Fractional Derivative

Let $f(x, t)$ be a function of two variables $x$ and $t$ defined in the positive quadrant of the $x t$-plane. The double Laplace transform of the function $f(x, t)$ as given by Sneddon [20] is defined by

$$
\begin{aligned}
L_{x} L_{t}\{f(x, t)\} & =\bar{f}(p, s) \\
& =\int_{0}^{\infty} e^{-p x} \int_{0}^{\infty} e^{-s t} f(x, t) d t d x,
\end{aligned}
$$

whenever that integral exists. Here $p$ and $s$ are complex numbers.

From this definition we deduce

$$
\begin{aligned}
L_{x} L_{t}[f(x) g(t)] & =\bar{f}(p) \bar{g}(s) \\
& =L_{x}[f(x)] L_{t}[g(t)] .
\end{aligned}
$$

The inverse double Laplace transform $L_{x}{ }^{-1} L_{t}{ }^{-1}\{\bar{f}(p, s)\}=$ $f(x, t)$ is defined as in $[21,22]$ by the complex double integral formula:

$$
\begin{aligned}
L_{x}^{-1} L_{t}^{-1}\{\bar{f}(p, s)\}=f(x, t) \\
\quad=\frac{1}{2 \pi i} \int_{c-i \infty}^{c+i \infty} e^{p x} d p \frac{1}{2 \pi i} \int_{d-i \infty}^{d+i \infty} e^{s t} \bar{f}(p, s) d s,
\end{aligned}
$$

where $\bar{f}(p, s)$ must be an analytic function for all $p$ and $s$ in the region defined by the inequalities $\operatorname{Re} p \geq c$ and $\operatorname{Re} s \geq d$, where $c$ and $d$ are real constants to be chosen suitably.

The double Laplace transform formulas for the partial derivatives of an arbitrary integer order as in [23] are

$$
\begin{aligned}
L_{x} L_{t}\left\{\frac{\partial^{m} f(x, t)}{\left.\partial x^{m}\right\}=} p^{m} \bar{f}(p, s)\right. & \\
& -\sum_{j=0}^{m-1} p^{m-1-j} L_{t}\left\{\frac{\partial^{j} f(0, t)}{\partial x^{j}}\right\}, \\
L_{x} L_{t}\left\{\frac{\partial^{n} f(x, t)}{\partial t^{n}}\right\}= & s^{n} \bar{f}(p, s) \\
& -\sum_{k=0}^{n-1} s^{n-1-k} L_{x}\left\{\frac{\partial^{k} f(x, 0)}{\partial t^{k}}\right\} .
\end{aligned}
$$

Definition 1. The Caputo fractional derivative of function $u(x, t)$ is defined in [18] as

$$
\begin{array}{r}
\frac{\partial^{\alpha} f(x, t)}{\partial x^{\alpha}}=\frac{1}{\Gamma(m-\alpha)} \int_{0}^{x}(x-\xi)^{m-\alpha-1} \frac{\partial^{m} f(\xi, t)}{\partial \xi^{m}} d \xi, \\
m-1<\alpha \leq m, m \in N, \\
\frac{\partial^{\beta} f(x, t)}{\partial t^{\beta}}=\frac{1}{\Gamma(n-\beta)} \int_{0}^{t}(t-\tau)^{n-\beta-1} \frac{\partial^{n} f(x, \tau)}{\partial \tau^{n}} d \tau, \\
n-1<\beta \leq n, n \in N .
\end{array}
$$

The double Laplace transform formulas for the partial fractional Caputo derivatives as in [23] are

$$
\begin{aligned}
L_{x} L_{t}\left\{\frac{\partial^{\alpha} f(x, t)}{\partial x^{\alpha}}\right\}= & p^{\alpha} \bar{f}(p, s) \\
& -\sum_{j=0}^{m-1} p^{\alpha-1-j} L_{t}\left\{\frac{\partial^{j} f(0, t)}{\partial x^{j}}\right\}, \\
L_{x} L_{t}\left\{\frac{\partial^{\beta} f(x, t)}{\partial t^{\beta}}\right\}= & s^{\beta} \bar{f}(p, s) \\
& -\sum_{k=0}^{n-1} s^{\beta-1-k} L_{x}\left\{\frac{\partial^{k} f(x, 0)}{\partial t^{k}}\right\} .
\end{aligned}
$$

Definition 2. The Mittag-Leffler function is defined by

$$
E_{\alpha, \beta}(t)=\sum_{k=0}^{\infty} \frac{t^{k}}{\Gamma(\alpha k+\beta)}, \quad t, \beta \in \mathbb{C}, \mathfrak{R}(\alpha)>0 .
$$

The single Laplace transform of the function $t^{\beta-1} E_{\alpha, \beta}\left(\lambda t^{\alpha}\right)$ takes the form

$$
L_{t}\left\{t^{\beta-1} E_{\alpha, \beta}\left(\lambda t^{\alpha}\right)\right\}=\frac{s^{\alpha-\beta}}{s^{\alpha}-\lambda}, \text { for }|\lambda|<\left|s^{\alpha}\right| .
$$

\section{Double Laplace Transform Method}

Consider the following general multiterms fractional telegraph equation as in [18]:

$$
\begin{aligned}
\frac{\partial^{\alpha} u(x, t)}{\partial x^{\alpha}}= & a \frac{\partial^{\beta} u(x, t)}{\partial t^{\beta}}+b \frac{\partial^{\gamma} u(x, t)}{\partial t^{\gamma}}+c u(x, t) \\
& +h(x, t), \\
& 1<\alpha, \beta \leq 2,0<\gamma \leq 1, x, t \geq 0,
\end{aligned}
$$

with initial conditions,

$$
\begin{aligned}
u(x, 0) & =f_{1}(x), \\
u_{t}(x, 0) & =f_{2}(x),
\end{aligned}
$$

and boundary conditions,

$$
\begin{gathered}
u(0, t)=g_{1}(t), \\
u_{x}(0, t)=g_{2}(t) .
\end{gathered}
$$

Here $a, b, c$ are constants and $h(x, t)$ is given function. 
Applying the double Laplace transform on both sides of (9), we get

$$
\begin{aligned}
p^{\alpha} \bar{u} & (p, s)-p^{\alpha-1} \bar{u}(0, s)-p^{\alpha-2} \overline{u_{x}}(0, s) \\
= & a\left[s^{\beta} \bar{u}(p, s)-s^{\beta-1} \bar{u}(p, 0)-s^{\beta-2} \overline{u_{t}}(p, 0)\right] \\
+ & b\left[s^{\gamma} \bar{u}(p, s)-s^{\gamma-1} \bar{u}(p, 0)\right]+c \bar{u}(p, s) \\
& +\bar{h}(p, s),
\end{aligned}
$$

where $\bar{h}(p, s)=L_{x} L_{t}\{h(x, t)\}$.

Further, applying single Laplace transform to initial (10) and boundary conditions (11), we get

$$
\begin{aligned}
\bar{u}(p, 0) & =\overline{f_{1}}(p), \\
\overline{u_{t}}(p, 0) & =\overline{f_{2}}(p), \\
\bar{u}(0, s) & =\overline{g_{1}}(s), \\
\overline{u_{x}}(0, s) & =\overline{g_{2}}(s) .
\end{aligned}
$$

By substituting (13) in (12) and simplifying, we obtain

$$
\begin{aligned}
& \bar{u}(p, s)=\frac{1}{\left(p^{\alpha}-a s^{\beta}-b s^{\gamma}-c\right)}\left[p^{\alpha-1} \overline{g_{1}}(s)\right. \\
& +p^{\alpha-2} \overline{g_{2}}(s)-a s^{\beta-1} \overline{f_{1}}(p)-a s^{\beta-2} \overline{f_{2}}(p) \\
& \left.\quad-b s^{\gamma-1} \overline{f_{1}}(p)+\bar{h}(p, s)\right] .
\end{aligned}
$$

Applying inverse double Laplace transform to (14), we obtain the solution of (9) in the form

$$
\begin{aligned}
& u(x, t)=L_{x}^{-1} L_{t}^{-1}\left[\frac { 1 } { ( p ^ { \alpha } - a s ^ { \beta } - b s ^ { \gamma } - c ) } \left[p^{\alpha-1} \overline{g_{1}}(s)\right.\right. \\
& +p^{\alpha-2} \overline{g_{2}}(s)-a s^{\beta-1} \overline{f_{1}}(p)-a s^{\beta-2} \overline{f_{2}}(p) \\
& \left.\left.\quad-b s^{\gamma-1} \overline{f_{1}}(p)+\bar{h}(p, s)\right]\right] .
\end{aligned}
$$

Here we assume that the inverse double Laplace transform of each term in the right side of (15) exists.

\section{Illustrative Examples}

In this section, we demonstrate the applicability of the previous method by giving examples.

Example 1. By substituting $a=1, b=1, c=1, \beta=2, \gamma=1$, and $h(x, t)=0$ in $(9)$,

$$
\begin{aligned}
& \frac{\partial^{\alpha} u(x, t)}{\partial x^{\alpha}}=\frac{\partial^{2} u(x, t)}{\partial t^{2}}+\frac{\partial u(x, t)}{\partial t}+u(x, t), \\
& 1<\alpha \leq 2, x, t \geq 0,
\end{aligned}
$$

subject to the initial and boundary conditions,

$$
\begin{aligned}
u(x, 0) & =f_{1}(x)=E_{\alpha}\left(x^{\alpha}\right)+x E_{\alpha, 2}\left(x^{\alpha}\right), \\
u_{t}(x, 0) & =f_{2}(x)=-\left[E_{\alpha}\left(x^{\alpha}\right)+x E_{\alpha, 2}\left(x^{\alpha}\right)\right], \\
u(0, t) & =g_{1}(t)=e^{-t} \\
u_{x}(0, t) & =g_{2}(t)=e^{-t}
\end{aligned}
$$

a homogeneous space-fractional telegraph equation.

Taking single Laplace transform to initial (17) and boundary conditions (18), we get

$$
\begin{aligned}
& \overline{f_{1}}(p)=\left(\frac{1}{p}+\frac{1}{p^{2}}\right) \frac{p^{\alpha}}{p^{\alpha}-1}, \\
& \overline{f_{2}}(p)=-\left(\frac{1}{p}+\frac{1}{p^{2}}\right) \frac{p^{\alpha}}{p^{\alpha}-1}, \\
& \overline{g_{1}}(s)=\overline{g_{2}}(s)=\frac{1}{s+1} .
\end{aligned}
$$

Substituting above in (15), we get solution of (16):

$$
\begin{aligned}
& u(x, t)=L_{x}^{-1} L_{t}^{-1}\left[\frac { 1 } { ( p ^ { \alpha } - s ^ { 2 } - s - 1 ) } \left[p^{\alpha-1} \frac{1}{s+1}\right.\right. \\
& +p^{\alpha-2} \frac{1}{s+1}-s\left(\frac{1}{p}+\frac{1}{p^{2}}\right) \frac{p^{\alpha}}{p^{\alpha}-1} \\
& \left.\left.+\left(\frac{1}{p}+\frac{1}{p^{2}}\right) \frac{p^{\alpha}}{p^{\alpha}-1}-\left(\frac{1}{p}+\frac{1}{p^{2}}\right) \frac{p^{\alpha}}{p^{\alpha}-1}\right]\right] .
\end{aligned}
$$

Simplifying, we obtain

$$
\begin{aligned}
& u(x, t)=L_{x}^{-1} L_{t}^{-1}\left[\frac{1}{(s+1)}\left(\frac{1}{p}+\frac{1}{p^{2}}\right) \frac{p^{\alpha}}{p^{\alpha}-1}\right], \\
& u(x, t)=e^{-t}\left[E_{\alpha}\left(x^{\alpha}\right)+x E_{\alpha, 2}\left(x^{\alpha}\right)\right],
\end{aligned}
$$

which agrees with the solution already obtained in [18].

If we take $\alpha=2$ then we get the exact solution of standard telegraph equation:

$$
u(x, t)=e^{x-t} .
$$

Example 2. By substituting $a=1, b=1, c=1, \beta=2, \gamma=1$, and $h(x, t)=-x^{2}-t+1$ in (9),

$$
\begin{aligned}
\frac{\partial^{\alpha} u(x, t)}{\partial x^{\alpha}}= & \frac{\partial^{2} u(x, t)}{\partial t^{2}}+\frac{\partial u(x, t)}{\partial t}+u(x, t)-x^{2}-t \\
& +1, \quad 1<\alpha \leq 2, x, t \geq 0,
\end{aligned}
$$

subject to the initial and boundary conditions,

$$
\begin{aligned}
u(x, 0) & =f_{1}(x) \\
& =x^{2}-2-2 x^{2} E_{\alpha, 3}\left(x^{\alpha}\right)+2 E_{\alpha, 1}\left(x^{\alpha}\right), \\
u_{t}(x, 0) & =f_{2}(x)=1, \\
u(0, t) & =g_{1}(t)=t, \\
u_{x}(0, t) & =g_{2}(t)=0,
\end{aligned}
$$

a space-fractional nonhomogeneous telegraph equation. 
Taking single Laplace transform to initial (24) and boundary conditions (25), we get

$$
\begin{aligned}
\overline{f_{1}}(p) & =\frac{2}{p^{3}}-\frac{2}{p}-2 \frac{p^{\alpha-3}}{p^{\alpha}-1}+2 \frac{p^{\alpha-1}}{p^{\alpha}-1} \\
& =\frac{2\left(p^{2}-1\right)}{p^{3}\left(p^{\alpha}-1\right)}, \\
\overline{f_{2}}(p) & =\frac{1}{p}, \\
\overline{g_{1}}(s) & =\frac{1}{s^{2}}, \\
\overline{g_{2}}(s) & =0 .
\end{aligned}
$$

Taking double Laplace transform of $h(x, t)$, we have

$$
\bar{h}(p, s)=\frac{-2}{p^{3} s}-\frac{1}{p s^{2}}+\frac{1}{p s} .
$$

Substituting above in (15), we get solution of (23):

$$
\begin{aligned}
& u(x, t)=L_{x}^{-1} L_{t}^{-1}\left[\frac { 1 } { ( p ^ { \alpha } - s ^ { 2 } - s - 1 ) } \left[p^{\alpha-1} \frac{1}{s^{2}}\right.\right. \\
& -s \frac{2\left(p^{2}-1\right)}{p^{3}\left(p^{\alpha}-1\right)}-\frac{1}{p}-\frac{2\left(p^{2}-1\right)}{p^{3}\left(p^{\alpha}-1\right)}-\frac{2}{p^{3} s}-\frac{1}{p s^{2}} \\
& \left.\left.+\frac{1}{p s}\right]\right] .
\end{aligned}
$$

Rearranging, we have

$$
\begin{aligned}
& u(x, t)=L_{x}^{-1} L_{t}^{-1}\left[\frac { 1 } { ( p ^ { \alpha } - s ^ { 2 } - s - 1 ) } \left[\left[\frac{p^{\alpha}}{p s^{2}}-\frac{1}{p}\right.\right.\right. \\
& \left.-\frac{1}{p s^{2}}-\frac{1}{p s}\right]+\left[\frac{-2 s\left(p^{2}-1\right)}{p^{3}\left(p^{\alpha}-1\right)}-\frac{2\left(p^{2}-1\right)}{p^{3}\left(p^{\alpha}-1\right)}\right. \\
& \left.\left.\left.-\frac{2}{p^{3} s}+\frac{2}{p s}\right]\right]\right] .
\end{aligned}
$$

Simplifying, we obtain

$$
\begin{aligned}
& u(x, t)=L_{x}^{-1} L_{t}^{-1}\left[\frac{1}{p s^{2}}+\frac{2\left(p^{2}-1\right)}{p^{3} s\left(p^{\alpha}-1\right)}\right], \\
& u(x, t)=t+x^{2}-2-2 x^{2} E_{\alpha, 3}\left(x^{\alpha}\right)+2 E_{\alpha, 1}\left(x^{\alpha}\right),
\end{aligned}
$$

which agrees with the solution already obtained in [18] for $\gamma=1$.

For $\alpha=2$, then $u(x, t)=t+x^{2}$.
Example 3. By substituting $a=1, b=1, c=0, \alpha=2, \gamma=1$, and $h(x, t)=-2 t\left(x^{2}-x\right)\left(t^{1-\beta} / \Gamma(3-\beta)+1\right)+2 t^{2}$ in (9),

$$
\begin{aligned}
& \frac{\partial^{\beta} u(x, t)}{\partial t^{\beta}}+\frac{\partial u(x, t)}{\partial t} \\
& =\frac{\partial^{2} u(x, t)}{\partial x^{2}}+2 t\left(x^{2}-x\right)\left(\frac{t^{1-\beta}}{\Gamma(3-\beta)}+1\right)-2 t^{2}, \\
& 1<\beta \leq 2, x, t \geq 0,
\end{aligned}
$$

subject to the initial and boundary conditions,

$$
\begin{gathered}
u(x, 0)=f_{1}(x)=0, \\
u_{t}(x, 0)=f_{2}(x)=0, \\
u(0, t)=g_{1}(t)=0, \\
u_{x}(0, t)=g_{2}(t)=-t^{2},
\end{gathered}
$$

a time fractional telegraph equation in [24].

Taking single Laplace transform to initial (32) and boundary conditions (33), we get

$$
\begin{aligned}
& \overline{f_{1}}(p)=\overline{f_{2}}(p)=\overline{g_{1}}(s)=0, \\
& \overline{g_{2}}(s)=\frac{-2}{s^{3}}
\end{aligned}
$$

Taking double Laplace transform of $h(x, t)$, we have

$$
\bar{h}(p, s)=-2\left(\frac{2}{p^{3}}-\frac{1}{p^{2}}\right)\left(\frac{1}{s^{3-\beta}}+\frac{1}{s^{2}}\right)+2 \frac{2}{p s^{3}} .
$$

Substituting above in (15), we get solution of (31):

$$
\begin{aligned}
& u(x, t)=L_{x}^{-1} L_{t}^{-1}\left[\frac { 1 } { ( p ^ { 2 } - s ^ { \beta } - s ) } \left[\frac{-2}{s^{3}}\right.\right. \\
& \left.\left.-2\left(\frac{2}{p^{3}}-\frac{1}{p^{2}}\right)\left(\frac{1}{s^{3-\beta}}+\frac{1}{s^{2}}\right)+2 \frac{2}{p s^{3}}\right]\right] .
\end{aligned}
$$

Simplifying, we obtain

$$
\begin{aligned}
& u(x, t)=L_{x}^{-1} L_{t}^{-1}\left[\left(\frac{2}{p^{3}}-\frac{1}{p^{2}}\right) \frac{2}{s^{3}}\right], \\
& u(x, t)=\left(x^{2}-x\right) t^{2} .
\end{aligned}
$$

Example 4. By substituting $a=1, b=1, c=1, \alpha=2$, and $h(x, t)=0$ in (9),

$$
\begin{aligned}
& \frac{\partial^{\beta} u(x, t)}{\partial t^{\beta}}+\frac{\partial^{\gamma} u(x, t)}{\partial t^{\gamma}}+u(x, t)=\frac{\partial^{2} u(x, t)}{\partial x^{2}}, \\
& 1<\beta \leq 2, \frac{1}{2}<\gamma \leq 1, x, t \geq 0,
\end{aligned}
$$

subject to the initial and boundary conditions,

$$
\begin{aligned}
u(x, 0) & =f_{1}(x)=0, \\
u_{t}(x, 0) & =f_{2}(x)=e^{x}, \\
u(0, t) & =g_{1}(t)=t E_{\beta-\gamma, 2}\left(-t^{\beta-\gamma}\right), \\
u_{x}(0, t) & =g_{2}(t)=t E_{\beta-\gamma, 2}\left(-t^{\beta-\gamma}\right),
\end{aligned}
$$

a homogeneous time fractional telegraph equation in [25]. 
Taking single Laplace transform to initial (39) and boundary conditions (40), we get

$$
\begin{aligned}
& \overline{f_{1}}(p)=0 \\
& \overline{f_{2}}(p)=\frac{1}{p-1}, \\
& \overline{g_{1}}(s)=\overline{g_{2}}(s)=\frac{s^{\beta-\gamma-2}}{s^{\beta-\gamma}+1} .
\end{aligned}
$$

Substituting above in (15), we get solution of (38):

$$
\begin{aligned}
& u(x, t)=L_{x}^{-1} L_{t}^{-1}\left[\frac { 1 } { ( p ^ { 2 } - s ^ { \beta } - s ^ { \gamma } - 1 ) } \left[p \frac{s^{\beta-\gamma-2}}{\left(s^{\beta-\gamma}+1\right)}\right.\right. \\
& \left.\left.+\frac{s^{\beta-\gamma-2}}{\left(s^{\beta-\gamma}+1\right)}-s^{\beta-2} \frac{1}{(p-1)}\right]\right] .
\end{aligned}
$$

Simplifying, we obtain

$$
\begin{aligned}
& u(x, t)=L_{x}^{-1} L_{t}^{-1}\left[\frac{1}{(p-1)} \frac{s^{\beta-\gamma-2}}{\left(s^{\beta-\gamma}+1\right)}\right], \\
& u(x, t)=e^{x} t E_{\beta-\gamma, 2}\left(-t^{\beta-\gamma}\right),
\end{aligned}
$$

which agrees with the solution already obtained in [25].

Example 5. By substituting $a=b=c=1, \alpha=2$, and $h(x, t)=$ $-\sinh x\left(t^{n} / \Gamma(n+1)\right)$ in $(9)$,

$$
\begin{aligned}
\frac{\partial^{\beta} u(x, t)}{\partial t^{\beta}}+\frac{\partial^{\beta-1} u(x, t)}{\partial t^{\beta-1}}+u(x, t) & \\
=\frac{\partial^{2} u(x, t)}{\partial x^{2}}+\sinh x \frac{t^{n}}{\Gamma(n+1)}, & \\
& 1<\beta \leq 2, x, t \geq 0,
\end{aligned}
$$

subject to the initial and boundary conditions,

$$
\begin{aligned}
u(x, 0) & =f_{1}(x)=0, \\
u_{t}(x, 0) & =f_{2}(x)=0, \\
u(0, t) & =g_{1}(t)=0, \\
u_{x}(0, t) & =g_{2}(t)=t^{n+\beta} E_{1, n+\beta+1}(-t),
\end{aligned}
$$

a nonhomogeneous time fractional telegraph equation in [25].

Taking single Laplace transform to initial (45) and boundary conditions (46), we get

$$
\begin{aligned}
& \overline{f_{1}}(p)=\overline{f_{2}}(p)=0, \\
& \overline{g_{1}}(s)=0, \\
& \overline{g_{2}}(s)=\frac{s^{-n-\beta}}{s+1} .
\end{aligned}
$$

Taking double Laplace transform of $h(x, t)$, we have

$$
\bar{h}(p, s)=\frac{-1}{\left(p^{2}-1\right) s^{n+1}} .
$$

Substituting above in (15), we get solution of (44):

$$
\begin{aligned}
& u(x, t)=L_{x}^{-1} L_{t}^{-1}\left[\frac { 1 } { ( p ^ { 2 } - s ^ { \beta } - s ^ { \beta - 1 } - 1 ) } \left[\frac{s^{-n-\beta}}{(s+1)}\right.\right. \\
& \left.\left.-\frac{1}{\left(p^{2}-1\right) s^{n+1}}\right]\right] .
\end{aligned}
$$

Simplifying, we obtain

$$
\begin{aligned}
& u(x, t)=L_{x}^{-1} L_{t}^{-1}\left[\frac{1}{\left(p^{2}-1\right)} \frac{s^{-n-\beta}}{(s+1)}\right], \\
& u(x, t)=(\sinh x) t^{n+\beta} E_{1, n+\beta+1}(-t),
\end{aligned}
$$

which agrees with the solution already obtained in [25].

Example 6. Consider the following space-fractional-order nonlinear telegraph equation:

$$
\begin{aligned}
& \frac{\partial^{\alpha} u(x, t)}{\partial x^{\alpha}}= \frac{\partial^{2} u(x, t)}{\partial t^{2}}+\frac{\partial u(x, t)}{\partial t}+u^{2}(x, t) \\
&-e^{-2 t}\left(x-x^{2}\right)^{2}-2 e^{-t} \frac{x^{2-\alpha}}{\Gamma(3-\alpha)}, \\
& 1<\alpha \leq 2, x, t \geq 0,
\end{aligned}
$$

under the initial conditions,

$$
u(x, 0)=-u_{t}(x, 0)=x-x^{2},
$$

and boundary conditions,

$$
\begin{gathered}
u(0, t)=0, \\
u_{x}(0, t)=e^{-t} .
\end{gathered}
$$

Applying the double Laplace transform on both sides of (51), we get

$$
\begin{aligned}
p^{\alpha} \bar{u} & (p, s)-p^{\alpha-1} \bar{u}(0, s)-p^{\alpha-2} \overline{u_{x}}(0, s) \\
= & s^{2} \bar{u}(p, s)-s \bar{u}(p, 0)-\overline{u_{t}}(p, 0)+s \bar{u}(p, s) \\
& -\bar{u}(p, 0)-\frac{2}{(s+1) p^{3-\alpha}} \\
& +L_{x} L_{t}\left[u^{2}(x, t)-e^{-2 t}\left(x-x^{2}\right)^{2}\right] .
\end{aligned}
$$

Further, applying single Laplace transform to initial (52) and boundary conditions (53), we get

$$
\begin{aligned}
& \bar{u}(p, 0)=-\overline{u_{t}}(p, 0)=\frac{1}{p^{2}}-\frac{2}{p^{3}}, \\
& \bar{u}(0, s)=0, \\
& \overline{u_{x}}(0, s)=\frac{1}{s+1} .
\end{aligned}
$$


By substituting (55) in (54) and simplifying, we obtain

$$
\begin{aligned}
& \left(p^{\alpha}-s^{2}-s\right) \bar{u}(p, s) \\
& =\frac{p^{\alpha}}{p^{2}(s+1)}-s\left[\frac{1}{p^{2}}-\frac{2}{p^{3}}\right]+\left[\frac{1}{p^{2}}-\frac{2}{p^{3}}\right] \\
& \quad-\left[\frac{1}{p^{2}}-\frac{2}{p^{3}}\right]-\frac{2 p^{\alpha}}{(s+1) p^{3}} \\
& \quad+L_{x} L_{t}\left[u^{2}(x, t)-e^{-2 t}\left(x-x^{2}\right)^{2}\right] \\
& \left(p^{\alpha}-s^{2}-s\right) \bar{u}(p, s) \\
& =\left[\frac{p^{\alpha}}{p^{2}(s+1)}-\frac{s}{p^{2}}\right]-\left[\frac{2 p^{\alpha}}{(s+1) p^{3}}-\frac{2 s}{p^{3}}\right] \\
& \quad+L_{x} L_{t}\left[u^{2}(x, t)-e^{-2 t}\left(x-x^{2}\right)^{2}\right] \\
& \bar{u}(p, s) \\
& \left.\quad+\frac{1}{p^{2}}-\frac{2}{p^{3}}\right] \frac{1}{(s+1)} \\
& \quad \frac{1}{\left.p^{\alpha}-s^{2}-s\right)} L_{x} L_{t}\left[u^{2}(x, t)-e^{-2 t}\left(x-x^{2}\right)^{2}\right] .
\end{aligned}
$$

Applying inverse double Laplace transform of (57), we get

$$
\begin{gathered}
u(x, t)=\left(x-x^{2}\right) e^{-t}+L_{x}^{-1} L_{t}^{-1}\left[\frac{1}{\left(p^{\alpha}-s^{2}-s\right)}\right. \\
\left.\cdot L_{x} L_{t}\left[u^{2}(x, t)-e^{-2 t}\left(x-x^{2}\right)^{2}\right]\right] .
\end{gathered}
$$

Now we apply the Iterative method as in [26];

$$
u(x, t)=\sum_{i=0}^{\infty} u_{i}(x, t) .
$$

Substituting (59) in (58), we get

$$
\begin{gathered}
\sum_{i=0}^{\infty} u_{i}(x, t)=\left(x-x^{2}\right) e^{-t}+L_{x}^{-1} L_{t}^{-1}\left[\frac{1}{\left(p^{\alpha}-s^{2}-s\right)}\right. \\
\left.\cdot L_{x} L_{t}\left[\left[\sum_{i=0}^{\infty} u_{i}(x, t)\right]^{2}-e^{-2 t}\left(x-x^{2}\right)^{2}\right]\right] .
\end{gathered}
$$

The nonlinear term $N$ is decomposed as

$$
\begin{aligned}
& {\left[\sum_{i=0}^{\infty} u_{i}(x, t)\right]^{2}} \\
& =\left[u_{0}(x, t)\right]^{2} \\
& \quad+\sum_{i=1}^{\infty}\left[\left[\sum_{k=0}^{i} u_{k}(x, t)\right]^{2}-\left[\sum_{k=0}^{i-1} u_{k}(x, t)\right]^{2}\right] .
\end{aligned}
$$

Substituting (61) in (60), we get

$$
\begin{aligned}
& \sum_{i=0}^{\infty} u_{i}(x, t)=\left(x-x^{2}\right) e^{-t}+L_{x}^{-1} L_{t}^{-1}\left[\frac{1}{\left(p^{\alpha}-s^{2}-s\right)}\right. \\
& \left.\cdot L_{x} L_{t}\left[\left[u_{0}(x, t)\right]^{2}-e^{-2 t}\left(x-x^{2}\right)^{2}\right]\right] \\
& +L_{x}^{-1} L_{t}^{-1}\left[\frac{1}{\left(p^{\alpha}-s^{2}-s\right)}\right. \\
& \left.\cdot L_{x} L_{t}\left[\sum_{i=1}^{\infty}\left[\left[\sum_{k=0}^{i} u_{k}(x, t)\right]^{2}-\left[\sum_{k=0}^{i-1} u_{k}(x, t)\right]^{2}\right]\right]\right]
\end{aligned}
$$

Then we define the recurrence relations as

$$
\begin{aligned}
& u_{0}(x, t)=\left(x-x^{2}\right) e^{-t} \\
& u_{1}(x, t)=L_{x}^{-1} L_{t}^{-1}\left[\frac { 1 } { ( p ^ { \alpha } - s ^ { 2 } - s ) } L _ { x } L _ { t } \left[\left[u_{0}(x, t)\right]^{2}\right.\right. \\
& \left.\left.-e^{-2 t}\left(x-x^{2}\right)^{2}\right]\right]=0 \\
& u_{2}(x, t)=L_{x}^{-1} L_{t}^{-1}\left[\frac{1}{\left(p^{\alpha}-s^{2}-s\right)}\right. \\
& \left.\cdot L_{x} L_{t}\left[\left[u_{0}(x, t)+u_{1}(x, t)\right]^{2}-\left[u_{0}(x, t)\right]^{2}\right]\right]=0, \\
& u_{3}(x, t)=L_{x}^{-1} L_{t}^{-1}\left[\frac{1}{\left(p^{\alpha}-s^{2}-s\right)}\right. \\
& \cdot L_{x} L_{t}\left[\left[u_{0}(x, t)+u_{1}(x, t)+u_{2}(x, t)\right]^{2}\right. \\
& \left.\left.-\left[u_{0}(x, t)+u_{1}(x, t)\right]^{2}\right]\right]=0
\end{aligned}
$$

and so on.

Therefore, we obtain the solution of (51) as follows:

$$
u(x, t)=e^{x-2 t} \text {. }
$$

This is the required exact solution of (51).

\section{Conclusion}

We have applied double Laplace transform to obtain exact solutions of linear/nonlinear space-time fractional telegraph equations. All of the examples considered show that double Laplace transform method is capable of reducing the volume of computational work as compared to other methods. It may be concluded that DLT technique solves the problems without using Adomian polynomials, Lagrange multiplier value, He's polynomials, and small parameters.

\section{Competing Interests}

The authors declare no competing interests regarding the publication of this paper. 


\section{References}

[1] E. Orsingher and Z. Xuelei, "The space-fractional telegraph equation and the related fractional telegraph process," Chinese Annals Mathematics B, vol. 24, no. 1, pp. 45-56, 2003.

[2] E. Orsingher and L. Beghin, "Time-fractional telegraph equations and telegraph processes with Brownian time," Probability Theory and Related Fields, vol. 128, no. 1, pp. 141-160, 2004.

[3] S. Momani, "Analytic and approximate solutions of the spaceand time-fractional telegraph equations," Applied Mathematics and Computation, vol. 170, no. 2, pp. 1126-1134, 2005.

[4] M. Garg and A. Sharma, "Solution of space-time fractional telegraph equation by Adomian decomposition method," Journal of Inequalities and Special Functions, vol. 2, no. 1, pp. 1-7, 2011.

[5] G. Adomian, Solving Frontier Problems of Physics: The Decomposition Method, vol. 60, Kluwer Academic Publishers, Dordrecht, The Netherlands, 1994.

[6] J. Chen, F. Liu, and V. Anh, "Analytical solution for the timefractional telegraph equation by the method of separating variables," Journal of Mathematical Analysis and Applications, vol. 338, no. 2, pp. 1364-1377, 2008.

[7] F. Huang, "Analytical solution for the time-fractional telegraph equation," Journal of Applied Mathematics, vol. 2009, Article ID 890158, 9 pages, 2009.

[8] J.-H. He, "Variational iteration method-a kind of non-linear analytical technique: some examples," International Journal of Non-Linear Mechanics, vol. 34, no. 4, pp. 699-708, 1999.

[9] A. Sevimlican, "An approximation to solution of space and time fractional telegraph equations by He's variational iteration method," Mathematical Problems in Engineering, vol. 2010, Article ID 290631, 10 pages, 2010.

[10] A. Yildirim, "He's homotopy perturbation method for solving the space- and time-fractional telegraph equations," International Journal of Computer Mathematics, vol. 87, no. 13, pp. 2998-3006, 2010.

[11] S. Das, K. Vishal, P. K. Gupta, and A. Yildirim, "An approximate analytical solution of time-fractional telegraph equation," Applied Mathematics and Computation, vol. 217, no. 18, pp. 7405-7411, 2011.

[12] M. Garg, P. Manohar, and S. L. Kalla, "Generalized differential transform method to space-time fractional telegraph equation," International Journal of Differential Equations, vol. 2011, Article ID 548982, 9 pages, 2011.

[13] L. Galue, "Solution of some fractional order telegraph equations," Revista Colombiana de Mathematicas, vol. 48, no. 2, pp. 247-267, 2014.

[14] V. K. Srivastava, M. K. Awasthi, and M. Tamsir, "RDTM solution of Caputo time fractional-order hyperbolic telegraph equation," AIP Advances, vol. 3, no. 3, 2013.

[15] Y. Khan, J. Diblik, N. Faraz, and Z. Smarda, "An efficient new perturbative Laplace method for space-time fractional telegraph equations," Advances in Difference Equations, vol. 2012, article 204, 2012.

[16] D. Kumar, J. Singh, and S. Kumar, "Analytic and approximate solutions of space-time fractional telegraph equations via laplace transform," Walailak Journal of Science and Technology, vol. 11, no. 8, pp. 711-728, 2014.

[17] A. Prakash, "Analytical method for space-fractional telegraph equation by homotopy perturbation transform method," Nonlinear Engineering, vol. 5, no. 2, pp. 123-128, 2016.
[18] F. A. Alawad, E. A. Yousif, and A. I. Arbab, "A new technique of Laplace variational iteration method for solving spacetime fractional telegraph equations," International Journal of Differential Equations, vol. 2013, Article ID 256593, 10 pages, 2013.

[19] S. Kumar, "A new analytical modelling for fractional telegraph equation via Laplace transform," Applied Mathematical Modelling, vol. 38, no. 13, pp. 3154-3163, 2014.

[20] I. N. Sneddon, The Use of Integral Transforms, Tata Mcgraw Hill, 1974.

[21] L. Debnath and D. Bhatta, Integral Transforms and Their Applications, CRC Press, Taylor \& Francis Group, Boca Raton, Fla, USA, 3rd edition, 2015.

[22] L. Debnath, "The double Laplace transforms and their properties with applications to functional, integral and partial differential equations," International Journal of Applied and Computational Mathematics, vol. 2, no. 2, pp. 223-241, 2016.

[23] A. M. Anwar, F. Jarad, D. Baleanu, and F. Ayaz, "Fractional Caputo heat equation within the double Laplace transform," Romanian Journal of Physics, vol. 58, no. 1-2, pp. 15-22, 2013.

[24] S. Sarwar and M. M. Rashidi, "Approximate solution of twoterm fractional-order diffusion, wave-diffusion, and telegraph models arising in mathematical physics using optimal homotopy asymptotic method," Waves in Random and Complex Media, vol. 26, no. 3, pp. 365-382, 2016.

[25] R. Joice Nirmala and K. Balachandran, "Analysis of solutions of time fractional telegraph equation," Journal of the Korean Society for Industrial and Applied Mathematics, vol. 18, no. 3, pp. 209-224, 2014.

[26] R. R. Dhunde and G. L. Waghmare, "Double Laplace transform combined with Iterative method for solving non-linear telegraph equation," Journal of the Indian Mathematical Society, vol. 83, no. 3-4, pp. 221-230, 2016. 


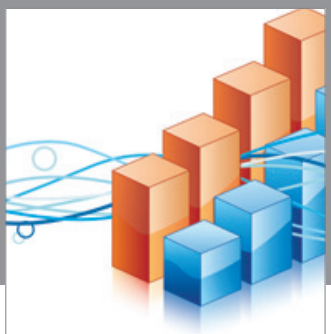

Advances in

Operations Research

vatem alat4

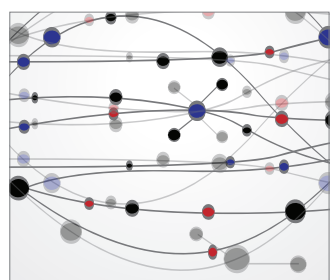

\section{The Scientific} World Journal
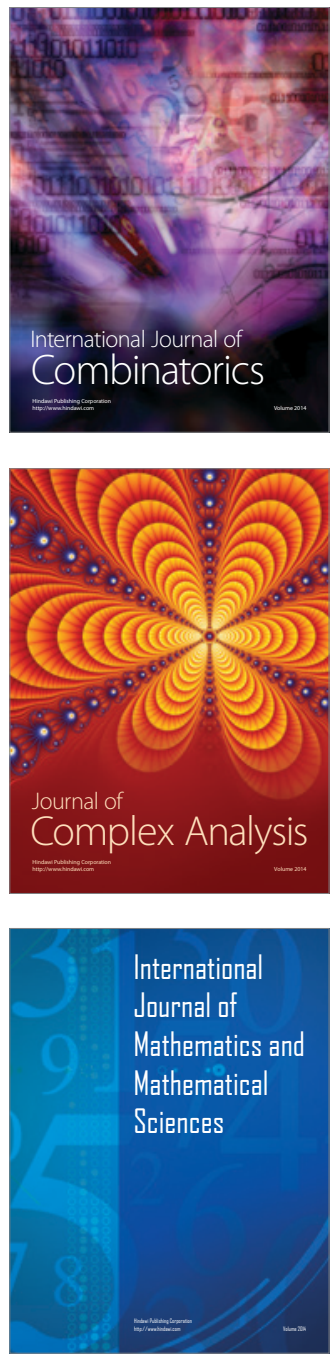
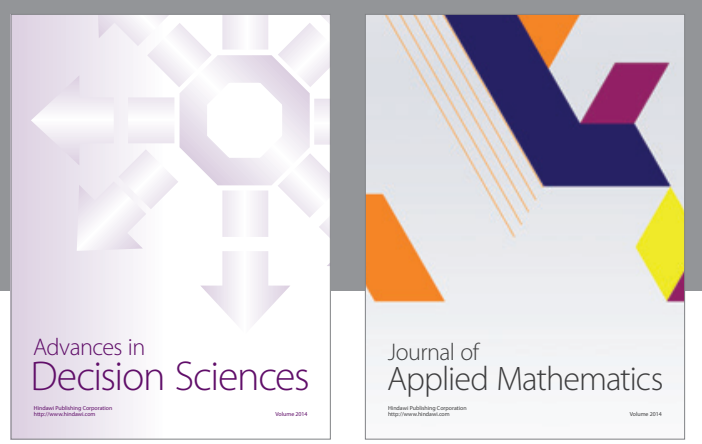

Algebra

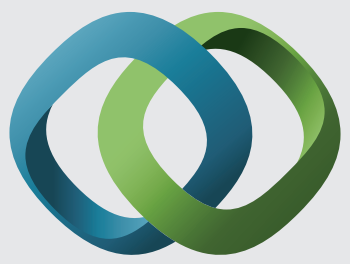

\section{Hindawi}

Submit your manuscripts at

http://www.hindawi.com
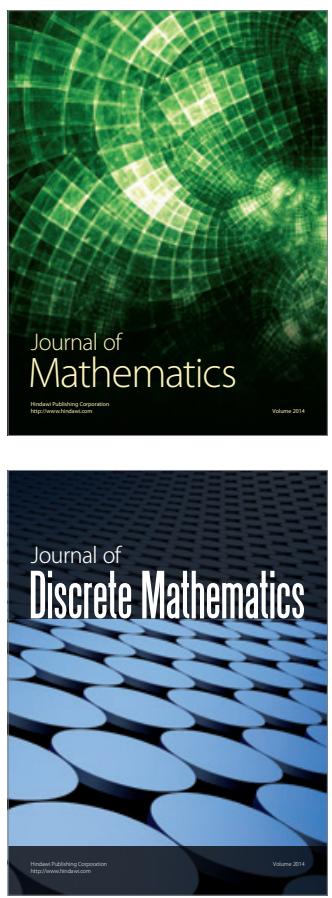

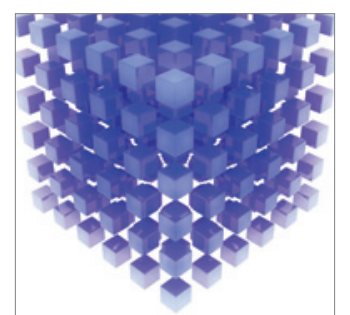

Mathematical Problems in Engineering
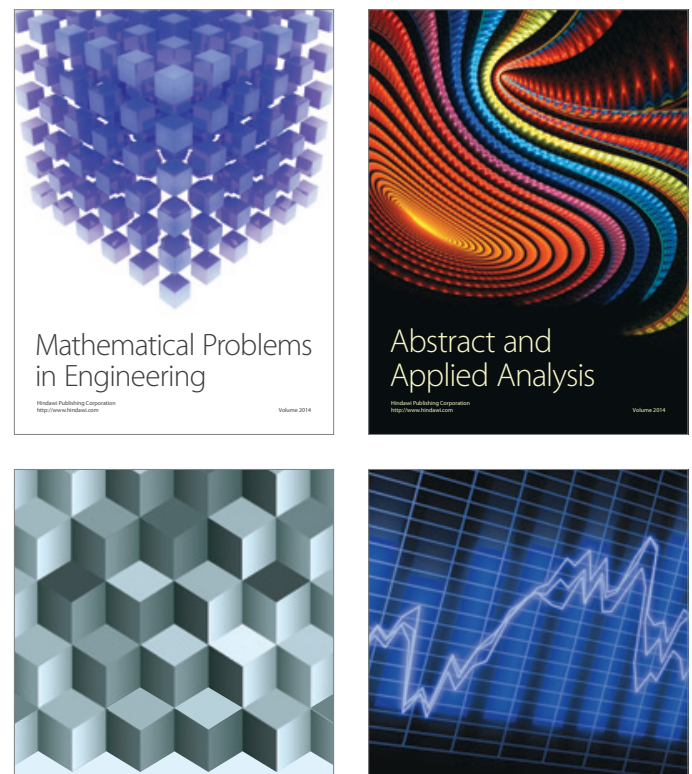

Journal of

Function Spaces

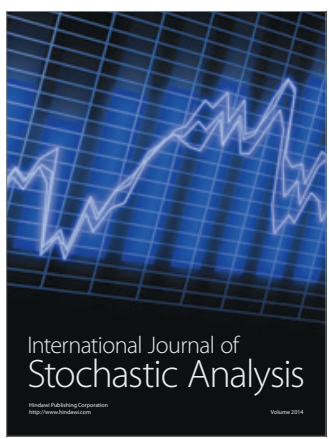

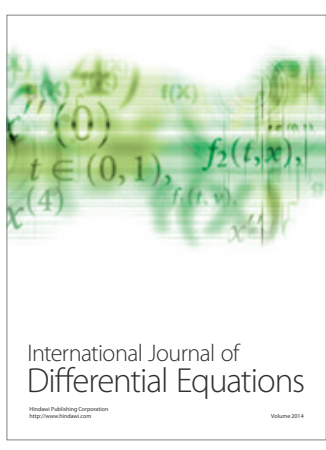
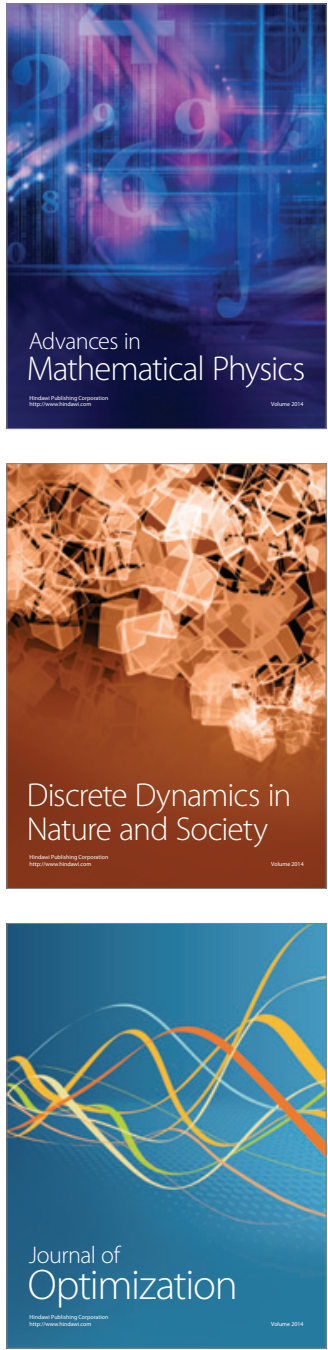